\title{
Multivalued Adaptive Neuro-Fuzzy Controller for Robot Vehicle
}

\author{
Giuliani Paulineli Garbi, Victor Orlando Gamarra Rosado, Francisco José Grandinetti \\ UNESP - Paulista State University, College of Engineering, Department of Mechanical Engineering. \\ Guaratinguetá - São Paulo - Brazil
}

\begin{abstract}
Robotic vehicle navigation in unstructured and uncertain environments is still a challenge. This paper presents the implementation of a multivalued neurofuzzy controller for autonomous ground vehicle (AGVs) in indoor environments. The control system consists of a hierarchy of mobile robot using multivalued adaptive neuro-fuzzy inference system behaviors.
\end{abstract}

Keywords: Mobile robot, Multivalued system, Adaptive neuro-fuzzy control.

\section{Introduction}

Robot vehicle appeared from growth exploration in unstructured and uncertain environments. These robots are becoming increasingly sophisticated and autonomous, and demand more intelligent behaviors from them in complex environments [7]. Among the applications currently it is like main the spatial exploration vehicles in Mars and vehicle unmanned used in special missions.

To achieve navigation expectations must address a set of problems associated with real world environments. The dynamics of the environments can be only partially predictive through partial and approximation knowledge of the environments, partial sensing and noisy, and an agent hardware execution represented by controller [2] and [7].

The controller complexity is overcome having a hierarchical architecture by decomposing the navigation control problem into several subsystems, which can be controlled independently and in parallel, will reduce the negative effect that a large rule-base may have on real time performance [4].

The subsystems and their controllers are known as reactive behaviors and this approach is known as robot behaviors. The concept of behavior control was initially seen as a special form of decentralized switching control, where a command fusion is used to combine the output of several individual behaviors subsystems [8] and [9].
However, this univalued structure has been found to have serious flaws. In particular, by treating behaviors as fully autonomous, this structure tends to cause the robot to be indecisive when the behaviors have mutually exclusive interests with nearly equal importance. This observation led to the introduction of multivalued behavior control system [1].

Multivalued fuzzy logic control is well suited for controlling the complex robot navigation because it is capable of represent degrees of truth on a numeric scale and making inferences even under uncertainty, which allows to express partial preferences and to combine them using logical operators [3].

Neural networks and others methods are used to solve the behavior learning problem. Learning allows autonomous robots to acquire knowledge by interacting with the environment and subsequently adapting their behavior and solve the problem of insufficient knowledge for designing the controller rule-base [5], [6] and [10].

This paper discusses with detailed an adaptive neuro-fuzzy inference system (ANFIS) implemented in structure of multivalued behaviors system for robotic vehicle navigation. The control system is organized in hierarchy of various adaptive neuro-fuzzy behaviors that represent physical position, where is implemented a multi-valued behavior control adaptive neuro fuzzy system to combine the degree preference output of several subsystems.

\section{Robotic Vehicle}

The rectangular shaped robotic vehicle has a differential driven platform steering system configured with two (dc) motors independently which control two wheels on a common axis and more two wheels swivel caster for support. The distance between wheels is $0.80 \mathrm{~m}$, the wheel radius is $0.008 \mathrm{~m}$, the wheel thickness is $0.05 \mathrm{~m}$ and robot speed is -0.1 to $0.3 \mathrm{~m} / \mathrm{s}$. The sense perception system consists of set electronic sensors to formation mobile robot behaviors. Each independent behavior uses the sensory information to determine it is course of action. The navigation control system requires range sensors and localization sensors. 
Ranger sensors are responsible to measure the distance of (potential) obstacles, consists of a total of seven infrared (IR) range sensors around the vehicle. Using infrared range sensors instead of sonar sensors allows avoiding some of the drawbacks that the later are usually suffering from multiple reflections.

These sensors are concerned to cover the whole field around the robotic vehicle, where three forward facing range sensors are used for front collision avoidance (F1, F2, F3), two lateral facing range sensors, right (R1, R2) and left (L1, L2) are used to control smooth contour following movements.

The main object of the navigation is to reach a goal position while avoiding obstacles. This information is provided by localization sensors. Global Positioning Systems (GPS), Inertial Navigation Systems (INS) and electronic compass are typical used localization sensors. The present robotic vehicle is equipped with a Global Positioning Systems (GPS) that measure the change in orientation of the vehicle. The direction of the mobile robotic is obtained between the relation of the actual position and the initial position. The physical position of the sensors shown in Fig. 1.

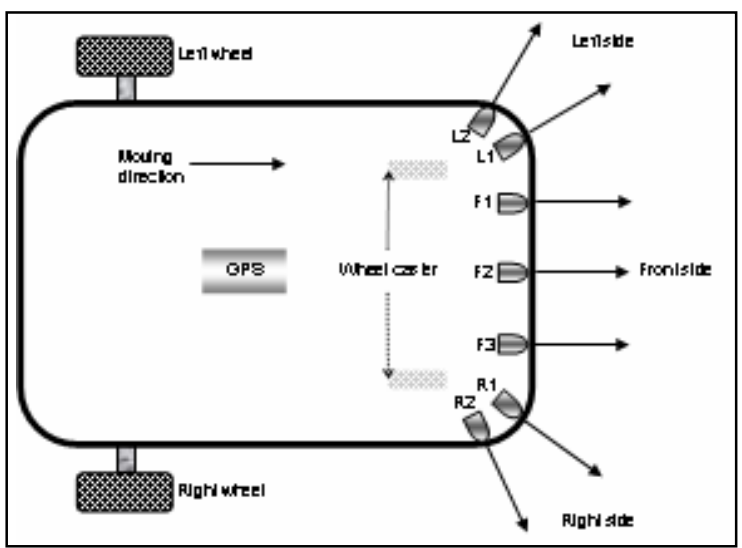

Fig. 1: Robotic vehicle’s sensors.

\section{Multivalued Adaptive Neuro- Fuzzy Behaviors Control System}

This section describes the design of multivalued adaptive neuro-fuzzy inference system (ANFIS) behaviors control system. A control schema, together with a set of object descriptor and a contextual condition, is packaged into a behavior. Behaviors play the role of situated actions: they indicated which movement should be performed which circumstances and with respect to which objects. Behaviors bridge the gap between abstract action description and physical control.
Multivalued architectures are systems where behaviors return their degree of preference to several control alternatives instead of a single control action. The preference for each alternative can then be evaluated to determine the best control action through. The hierarchical organization of the behaviors used for the control action of the robotic vehicle is show in Fig. 2.

The control action system obtains the speed for the robotic vehicle. In practice are two systems, one for each independent motor, because the adaptive neuro-fuzzy inference systems (ANFIS) used a Takagi/Sugeno type fuzzy inference system that allows a single output. ANFIS is a network type structure similar to that of a neural network, which through maps inputs and output membership functions and associated parameters can be used to interpret the input/output map. The parameters associated with the input membership functions will change through the learning process.

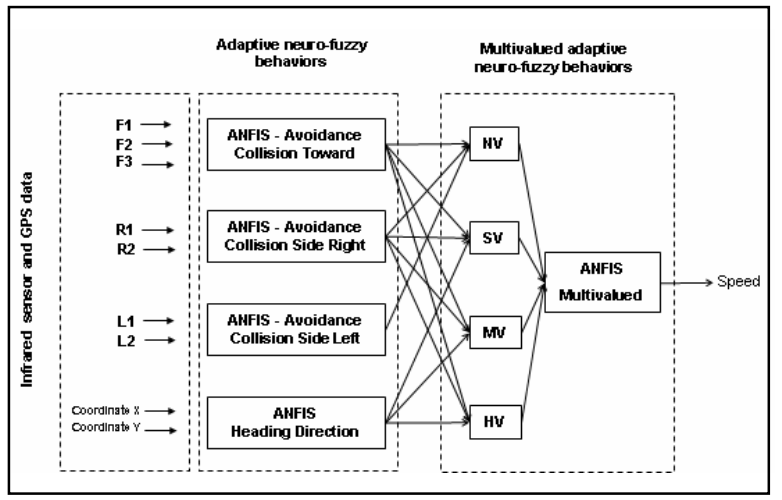

Fig. 2: Architecture of multivalued adaptive neuro-fuzzy behaviors system to the speed activity.

The computation of these parameters is facilitated by a gradient vector, which provides a measure of how well the fuzzy inference system (FIS) is modeling the input/output data for a given set of parameters. Once the gradient vector is obtained, any of several optimization routines could be applied in order to adjust the parameters so as to reduce some error measure. ANFIS uses either backpropagation or a combination of least squares estimation and backpropagation for membership function parameter estimation.

The fuzzy rules for each behavior are obtained through model validation process, which the input vectors from input/output data sets on the FIS was not trained, are presented to the trained FIS model. When checking data is presented to ANFIS as well as training data, the FIS model is selected to have 
parameters associated with the minimum checking data model error.

The speed control is achieved using four behaviors: Avoidance Collision Toward, Avoidance Collision Side Right and Avoidance Collision Side Left that use range finding sensors to determine distances to the nearest (potential) obstacles; and Heading Direction that use Global Positioning Systems (GPS) to determine the direction of the robotic vehicle through coordinate $\mathrm{X}$ and $\mathrm{Y}$.

The control command for the speed activity is the speed preference degree applied independently to the motors represented by three fuzzy sets: Stop Speed (SS), Medium Speed (MS) and High Speed (HS). With to intend to represent the motors velocity fuzzy sets are used a Gaussian membership was the simplest and best suited for this case, where $\mu \varepsilon(0,1)$ is the preference degree.

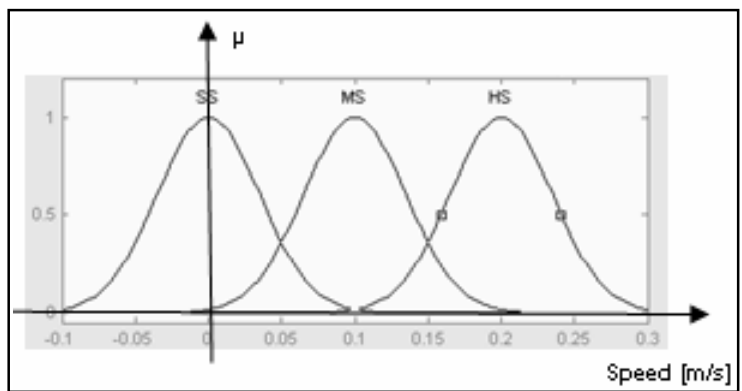

Fig. 3: Speed fuzzy sets representation.

\subsection{Avoidance Collision Toward Behaviors}

The control action system obtains the speed for the robotic vehicle, one for each independent motor. To represent the development of the ANFIS behaviors, this section show the control action for right motor speed, because ANFIS Model Structure, input membership functions, fuzzy rules, training data set and checking data set are identical to left motor speed.

The Avoidance Collision Toward behavior have ANFIS Model Structure with 3 inputs sensors F1, F2, F3 and 1 output represented the speed preference degree. The ANFIS Model Structure is shown in Fig. 4.

The obstacle is detected by three sensors, Fig. 4 . The range data provided are fed to ANFIS behaviors, which calculate the speed right motor preference. The input membership function is the distance to the obstacle with the corresponding fuzzy set (near, medium, away).

The data set used for training Avoidance Collision Toward behaviors ANFIS, consisted of 47 measurements data points, which range sensors F1, F2,

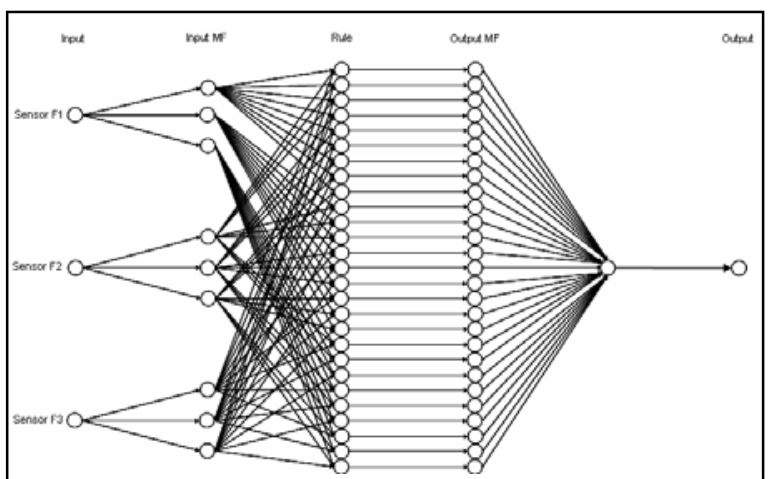

Fig. 4: ANFIS Model Structure for Avoidance Collision Toward to right motor speed.

F3 and the preference right motor speed, and used a checking data set obtained by applying a $5 \%$ random noise to the training data set. A combination of least squares estimation and backpropagation errors of the output over 50 training epochs are plotted in Fig. 5.

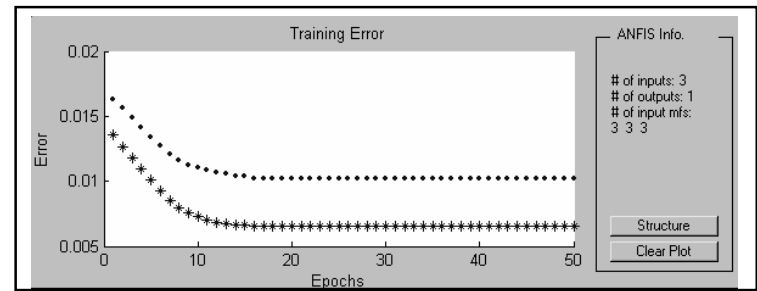

Fig. 5: Plot errors during the training Avoidance Collision Toward behavior to right motor speed.

\subsection{Avoidance Collision Side Right and Left}

The Avoidance Collision Side Right and Avoidance Collision Side Left behaviors have the same ANFIS Model Structure, with 2 inputs, respective sensors R1, R2 and L1, L2 and 1 output represented the speed preference degree. The ANFIS Model Structure is shown in Fig. 6.

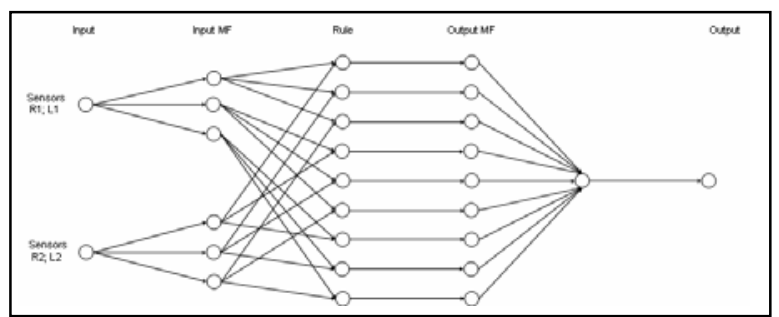

Fig. 6: ANFIS Avoidance Collision Side Right and Left behaviors to right motor speed. 


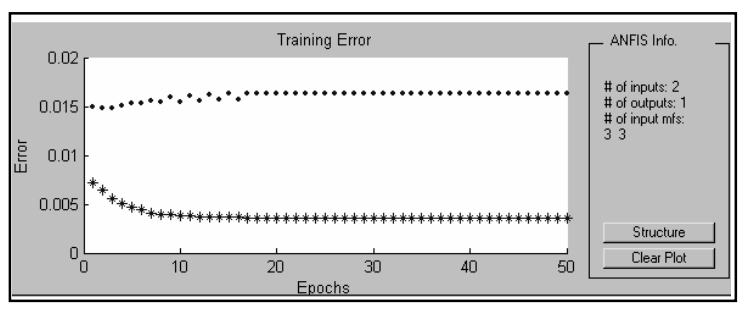

Fig. 7: Plot errors during the training Avoidance Collision Side Right behavior to right motor speed.

Two sensors detect the obstacle, as Fig. 6. The range data provided are fed to ANFIS behaviors, which calculate the speed right motor preference. The input membership function is the distance to the obstacle with the corresponding fuzzy set (near, medium, away). The data set used for training Avoidance Collision Side Right behaviors ANFIS, consisted of 26 measurements data points which range sensors R1, R2 and the preference right motor speed, and used a checking data set obtained by applying a $5 \%$ random noise to the training data set. A combination of least squares estimation and backpropagation errors of the output over 50 training epochs are plotted in Fig. 7.

\subsection{Heading Direction Behaviors}

The Heading Direction behaviors ANFIS Model Structure is formatted with 2 inputs coordinate $\mathrm{X}$ and $\mathrm{Y}$ respectively and 1 output represented the speed preference degree. The ANFIS Model Structure is shown in Fig. 8.

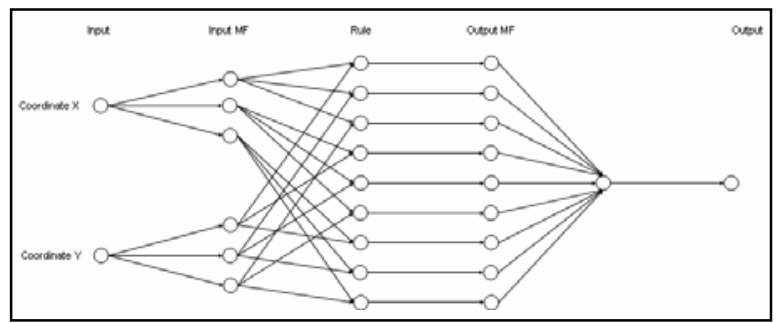

Fig. 8: ANFIS Heading Direction behavior to right motor speed.

The actual position detected by GPS data provided are fed to ANFIS behaviors, which calculate the speed right motor preference to determine the direction of the robotic vehicle. The input membership function to coordinate $\mathrm{X}-\mathrm{Y}$ is the distance to the origin with the corresponding fuzzy set (away target, medium target, near target).

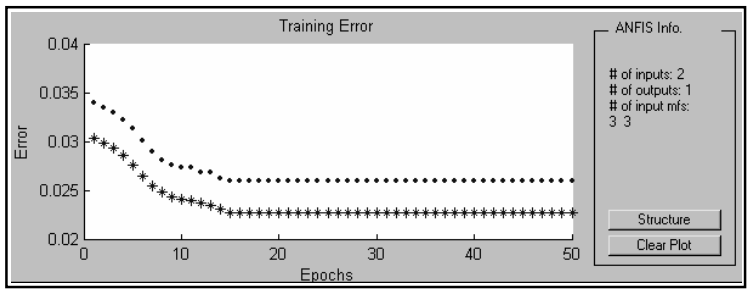

Fig. 9: Plot errors during the training Avoidance Heading Direction behavior to right motor speed.

The data set used for training Heading Direction behaviors ANFIS, consisted of 47 measurements data points, which range coordinate $\mathrm{X}, \mathrm{Y}$ and the preference right motor speed, and used a checking data set obtained by applying a $5 \%$ random noise to the training data set. A combination of least squares estimation and backpropagation errors of the output over 50 training epochs are plotted in Fig. 9.

\subsection{Multivalued Adaptive Neuro- Fuzzy Behaviors}

The Multivalued Adaptive Neuro-Fuzzy behavior calculated the vehicle speed during the trajectory avoidance obstacle and reaching the target. The avoidance collision is detected by Avoidance Collision Toward, Side Right and Side Left behaviors, and the target position is obtained by Heading Direction behavior. The speed preference degrees calculated by each behavior are fed to ANFIS Multivalued behavior, which calculated the motors speed.

The resulting membership functions for the Avoidance Collision Toward, Avoidance Collision Side Right, Side Left and Heading Direction behaviors inputs shown in Fig. 12. Was experimented several types membership functions, the Gaussian membership functions to be best suited for this case and to all behaviors. The general form of a fuzzy rule for a 4 input and 1 output first order Takagi/Sugeno type controller is:

$$
\begin{aligned}
& \text { IF x is } A_{a} \text { AND y is } B_{b} \text { AND } z \text { is } C_{c} \text { AND wis } D_{d} \\
& \text { THEN } \\
& F_{L}=p_{L} x+q_{L} y+r_{L} z+s_{L} w+t_{L} ; \\
& \text { for } a=1, \ldots, F ; b=1, \ldots, G ; c=1, \ldots, H ; d=1, \ldots, I ; \\
& L=1, \ldots, R ; R=F x G \times H x I
\end{aligned}
$$

Where $x, y, z$ and $w$ are the linguistic variables, $F_{L}$ is the output for $L$-th rules, $F, G, H$ and $I$ are the size of the fuzzy sets for the behaviors $A, B, C$ and $D$ and $R$ is the size of the rule base.

For Multivalued Adaptive Neuro-Fuzzy, behavior input $A, B, C$ and $D$ are the preference speed with 
corresponding fuzzy set (Stop Speed, Medium Speed, High Speed), and $R=81$ is the size of the rule base. The ANFIS Model Structure is shown in Fig. 10.

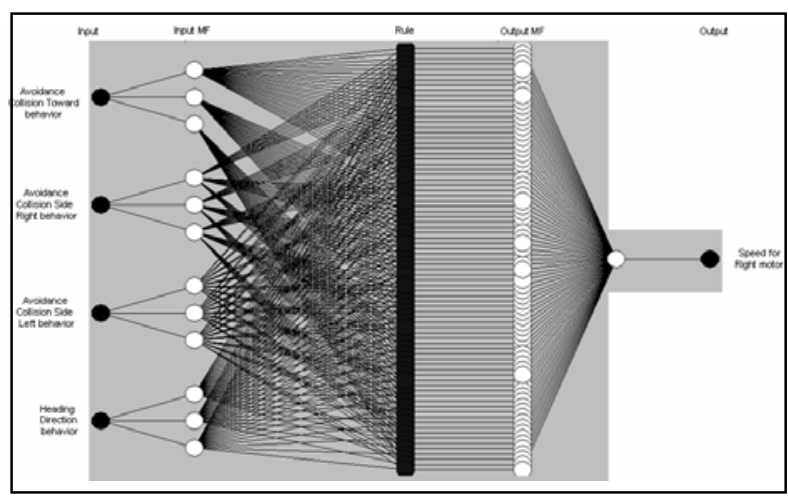

Fig. 10: ANFIS Model Structure for Multi-valued behaviors to right motor speed.

The speed preference data provided are fed to ANFIS behaviors, which calculate the speed right motor. The input membership function is the speed right motor with the corresponding fuzzy set Stop Speed (SS), Medium Speed (MS) and High Speed (HS), Fig. 12.

The data set used for training Multivalued behaviors ANFIS, consisted of 112 measurements data points, which speed preferences of the behaviors and the preference right motor speed, and used a checking data set obtained by applying a 5\% random noise to the training data set. A combination of least squares estimation and backpropagation errors of the output over 50 training epochs are plotted in Fig. 11.

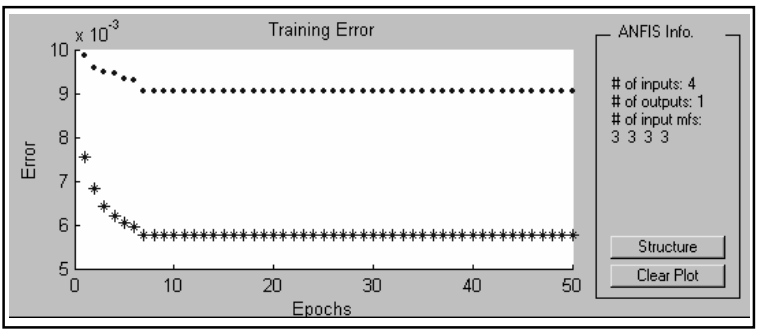

Fig. 11: Plot errors during the training Multi-valued behavior to right motor speed.

\section{Implementation and Performance Multivalued Adaptive Neuro-Fuzzy Controller}

This section presents a sample of the experimental results that show the performance of the proposed multivalued adaptive neuro-fuzzy control system, the experimental results are simulated on MatLab 6.5 toolbox software. The simulations are divided in three different scenarios. The control system is illustrated in Fig. 13.

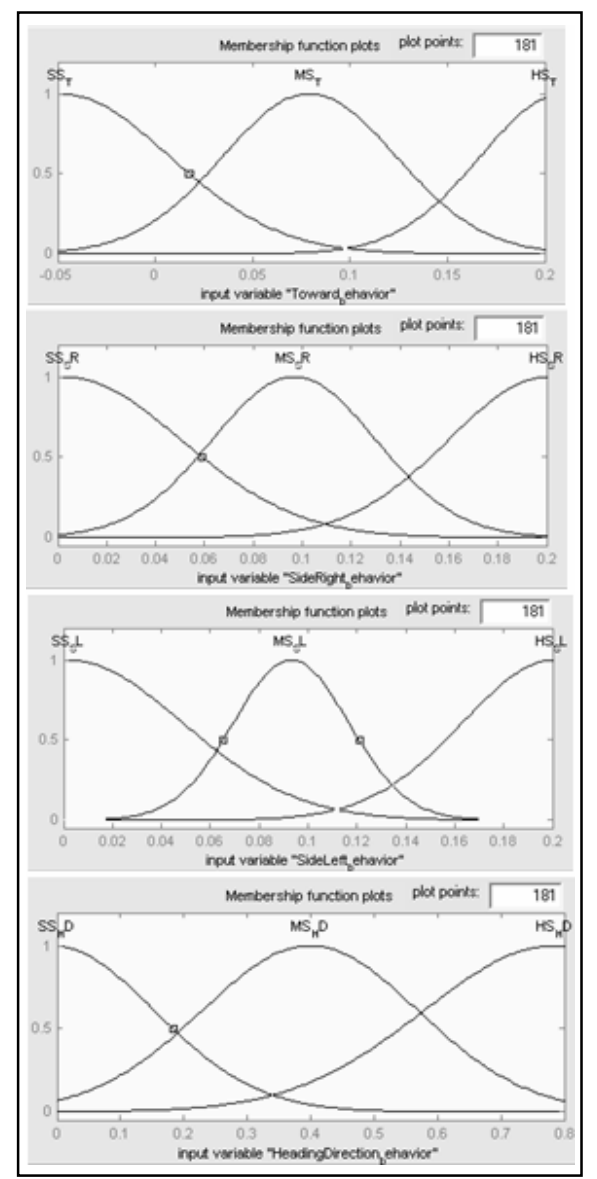

Fig. 12: Membership functions for the four inputs of ANFIS Multi-valued behavior to right motor speed; the measurement unit is the $\mathrm{m} / \mathrm{s}$.

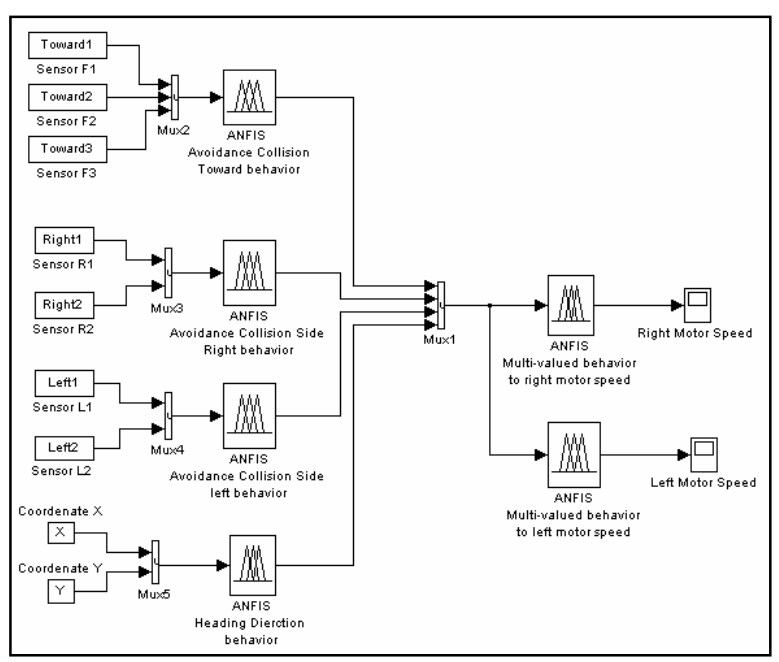

Fig. 13: ANFIS Controller system for robotic vehicle navigation. 


\subsection{Scenario I}

With some obstacle between the robot start position, coordinate $(0 ; 0)$, and the target position, coordinate $(4.7 ; 3.5)$. The robotic vehicle reaches the target position avoidance the obstacle during the trajectory by going to turn the obstacle. The scenario 1 is showed in Fig. 14, and the independent motor speed is showed in Fig. 15.

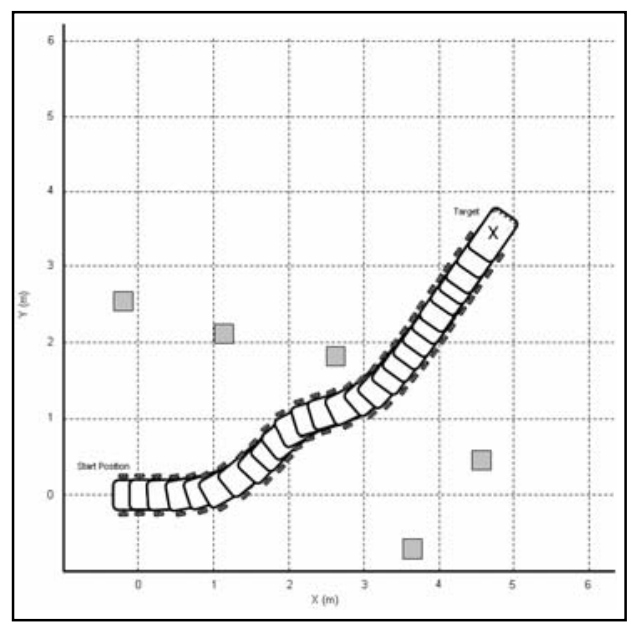

Fig. 14: Scenario I.

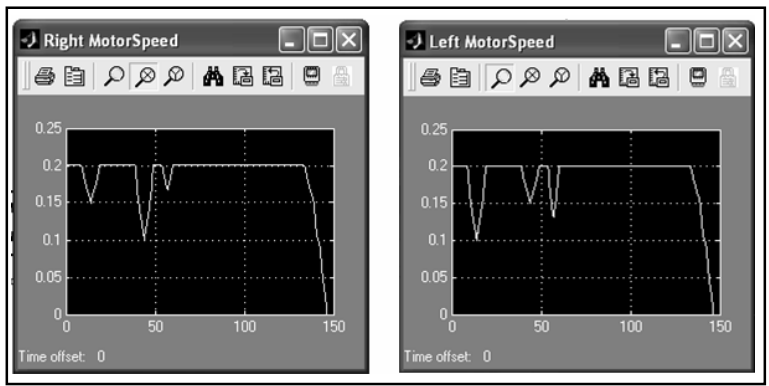

Fig. 15: Motor speed to scenario I.

\subsection{Scenario II}

With many obstacles between the robot start position, coordinate $(0 ; 0)$ and the target position, coordinate (4.1; 5.1). The robotic vehicle reaches the target position avoidance the obstacle during the trajectory by going to turn the obstacle. The Scenario 2 is showed in Fig. 16, and the independent motor speed is showed in Fig. 17.

\subsection{Scenario III}

Represent more complex situations that illustrate the ability to navigate very obstacles between the robot

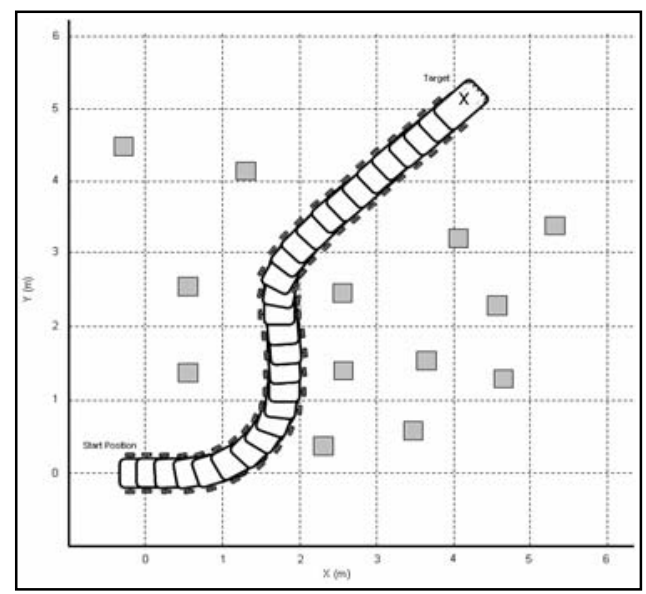

Fig. 16: Scenario II.

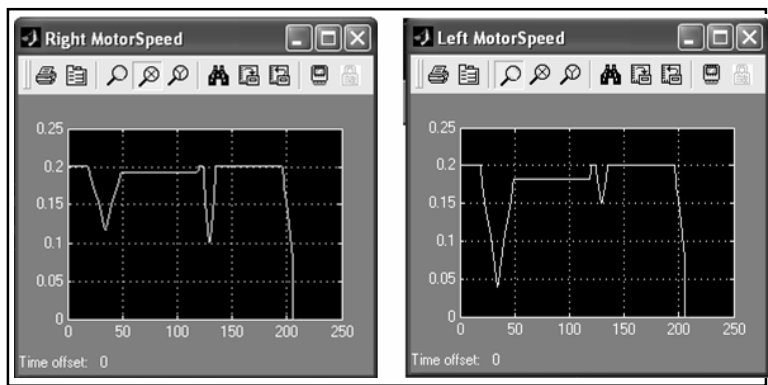

Fig. 17: Motor speed to scenario II.

start position coordinate $(0 ; 0)$ and the target position $(1.3 ; 4.3)$. The robotic vehicle reaches the target position avoidance the obstacle during the trajectory by going to turn the obstacle. The Scenario 3 is showed in Fig. 18, and the independent motor speed is showed in Fig. 19.

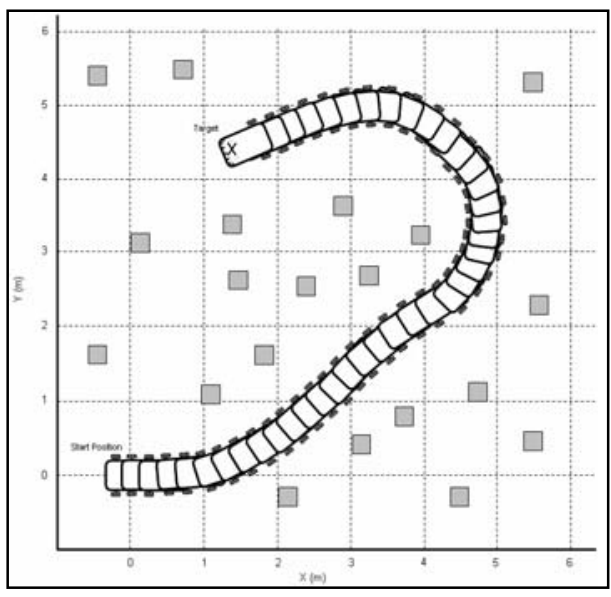

Fig. 18: Scenario III. 


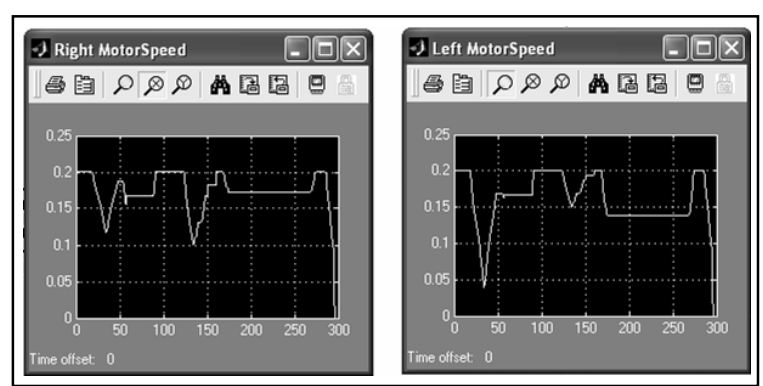

Fig. 19: Motor speed to scenario III.

\section{Conclusions}

This paper has discussed the major improvements done to implement the proposed multivalued adaptive neuro-fuzzy behavior system for robotic vehicle navigation. The simulation of the control system was implemented on MatLab 6.5 toolbox in different environments showing good performance in avoiding obstacles and reaching a target in a variety of obstacles configuration.

The success must be attributed to its adaptive neuro-fuzzy hierarchical architecture, which the learning allows robotic vehicle to acquire knowledge by interacting with the environment and subsequently adapting their behavior and solve the problem of insufficient knowledge for designing the ANFIS behaviors controllers rule-base, and allows all behaviors to express their in the available commands and fuse them by a fuzzy inference operation.

\section{References}

[1] M. F. Selekwa, D. D. Dunlap and E. G. Collins, Jr., Implementation of multi-valued fuzzy behavior control for robot navigation in cluttered, in Proceedings of IEEE International Conference on Robotics and Automation, Barcelona, Spain, pp. 3699-3706, April, 2005.

[2] P. Rusu, E. M. Petriu, T. E. Whalen, A. Cornell and H. J. W. Spoelder, Behavior-based neurofuzzy controller for mobile robot navigation, IEEE Trans. Instrum. Measur., 52(4):1335-1340, Aug, 2003.

[3] A. Saffiotti, K. Konolige and H. Ruspini, A multivalued logic approach to integrating planning and control, Artificial Intelligence, 76:481-526, 1995.

[4] R.C. Arkin, Behavior-Based Robotics, Cambridge Massachusetts: MIT Press, 1998.

[5] M. Figueiredo and F. Gomile, Design of fuzzy system using neuro-fuzzy net-works, IEEE Trans. Neural Networks, 10:815-827, Aug, 1999.
[6] J.S.R. Jang, ANFIS; Adaptive-network-based fuzzy inference system, IEEE Trans. Syst, Man., Cybern., 23: 665-685, June, 1993.

[7] R. Brooks, A robust layered control system for mobile robot, IEEE J. Robot. Automat. 2:14-23, Feb, 1986.

[8] E.W. Tunstel, Jr., Mobile robot autonomy via hierarchical fuzzy behavior control, in Proceedings of the 6th International Symposium on Robotics and Manufacturing, Montpellier, France, pp. 837-842, May, 1996.

[9] M.F. Selekwa and E. G. Collins, Jr., A centralized fuzzy behavior control for robot navigation, Proceedings of IEEE International Symposium on Intelligent control, Houston, Texas, pp. 602-607, October, 2003.

[10] S.K. Pradhan, D. R. Parhi and A. K. Panda, Navigation of multiple mobile robots using rulebased-neuro-fuzzy technique, in Proceedings of International Journal of Computational Intelligence, 3(2):142-151, 2006. 